\title{
Global Analysis of an SEIR Epidemic Model with Infectious Force under Intervention Strategies
}

\author{
Minmin Zhou, Tiansi Zhang \\ College of Science, University of Shanghai for Science and Technology, Shanghai, China \\ Email: 1447512612@qq.com
}

How to cite this paper: Zhou, M.M. and Zhang, T.S. (2019) Global Analysis of an SEIR Epidemic Model with Infectious Force under Intervention Strategies. Journal of Applied Mathematics and Physics, 7, 1706-1717.

https://doi.org/10.4236/jamp.2019.78117

Received: July 21, 2019

Accepted: August 13, 2019

Published: August 16, 2019

Copyright $\odot 2019$ by author(s) and Scientific Research Publishing Inc. This work is licensed under the Creative Commons Attribution International License (CC BY 4.0).

http://creativecommons.org/licenses/by/4.0/

\begin{abstract}
In this paper, we investigate the global stability of an SEIR (Susceptible-Exposed-Infected-Remove) epidemic model with infectious force under intervention strategies. To address this issue, we prove that the basic reproduction number $R_{0}$ plays an essential role in determining whether the disease extincts or persists. If $R_{0} \leq 1$, there is a unique disease-free equilibrium point of the model which is globally asymptotically stable and the disease dies out, and if $R_{0}>1$, there exists a unique endemic equilibrium point which is globally asymptotically stable and the disease persists.
\end{abstract}

\section{Keywords}

SEIR Epidemic Model, Intervention Strategies, Basic Reproduction Number, Global Stability

\section{Introduction}

As we all know, infectious disease has been ravaging human beings for thousands of years. In the long history, infectious disease has brought many disasters to human beings. For a long time, people have been fighting various infectious disease, and many methods have been used to study the spread of infectious disease, such as to control and eliminate infectious disease. Since the pioneer work of Kermack and McKendrick [1], mathematical models have been contributing to improve our understanding of infectious disease dynamics and helping us develop preventive measures to control infection spread qualitatively and quantitatively [2] [3]. The effect of intervention strategies, such as border screening, mask wearing, quarantine, isolation or communications through the mass media, 
plays an important role in administering efficient interventions to control disease spread and hopefully eliminate epidemic disease [4]-[9].

In recent years, a number of mathematical models have been formulated to describe the impact of intervention strategies on the dynamics of infectious disease [4]-[11]. Tang and Xiao indicate that strict interventions have been taken in mainland of China to slow down the initial spread of the disease [10] [12], and awareness through media and education plays an important role in changing behavior or contact patterns, and hence in limiting the spread of infectious disease [9].

In particular, Wang [4] formulates and analyzes an SIRS (Susceptible-Infected-Remove-Susceptible) epidemic model to study the impact of intervention strategies on the spread infectious disease and find that intervention strategies decrease endemic levels and tend to simplify diseases. In this article, we analyze the SEIR epidemic model to study the impact of intervention strategies on the spread of infectious disease.

In real life, epidemic tends to have an incubation period, as susceptible to infection after contacting with infected people. First of all, carrying virus, the virus is not immediately, but after a period of time, to onset and into the herd of infected people. In this paper, we mainly focus on the global stability analysis of the steady states for an SEIR epidemic model with infectious force under intervention.

We consider the global properties of this SEIR model and show that if the basic reproduction number $R_{0} \leq 1$, the disease-free equilibrium point is globally asymptotically stable, while if $R_{0}>1$, the disease-free equilibrium point is unstable and the unique endemic equilibrium point is globally asymptotically stable.

The rest organization of this article is arranged as follows: In Section 2, we present the model. In Section 3, we illustrate the main results and proof the main results in details. In Section 4, we provide the application of the results to SEIR model with infection force under intervention policy to support our findings. In the last section, we provide a brief discussion and summary of the results.

\section{Model Derivations}

We propose a deterministic SEIR epidemic model with infectious force. The model is given by

$$
\left\{\begin{array}{l}
\frac{\mathrm{d} S}{\mathrm{~d} t}=A-\mu S-H(I) S, \\
\frac{\mathrm{d} E}{\mathrm{~d} t}=H(I) S-(\mu+q) E, \\
\frac{\mathrm{d} I}{\mathrm{~d} t}=q E-(\mu+\delta+\gamma) I, \\
\frac{\mathrm{d} R}{\mathrm{~d} t}=\gamma I-\mu R,
\end{array}\right.
$$


where $S, E, I$ and $R$ denote the number of susceptible, exposed, infective and recovered individuals at time $t$, respectively, and $N(t)=S(t)+E(t)+I(t)+R(t)$. All parameters are positive with

$A$ : the recruitment rate of the population;

$\mu$ : the nature death rate of the population;

$q$ : the constant rate such that the exposed individuals become infective;

$\delta$ : the disease inducing death rate;

$\gamma:$ the natural recovery rate of the infective individuals.

The infective force $H(I)$ in (1) is a function of infective individuals which plays a key role in determining the transmission of disease. There are several different nonlinear transmission functions proposed by researchers, see more details, we refer to [4] [5] [13] [14] and the reference therein.

Model (1) includes the adaption of individuals behavior under intervention polices. For example, $H(I)$ may decrease as the number of infective individuals increase due to the fact that the population may tend to reduce the number of contacts per unit time under intervention polices. This has been interpreted as the psychological effect [5]. Mathematically, this phenomenon can be modeled as the infection force $H(I)$ which is increasing when $I$ is small and decreasing when $I$ is large. For simplicity in notations, we suppose that the infection force $H(I)$ can be factorized into $\frac{\beta I}{f(I)}$, where $\frac{1}{f(I)}$ represents the effect of intervention strategies on the reduction of valid contact coefficient $\beta$ [4]. It is worthy to note that, in the absence of intervention strategies, i.e. $f(I)=1$, the incidence rate becomes the well-known bilinear transmission rate $\beta S I$. To ensure a nonmonotonic infection force, we make the following assumptions [2]:

(H1) $f(0)>0$ and $f^{\prime}(I)>0$ for $I>0$.

(H2) There is $\zeta$ such that $\left(\frac{I}{f(I)}\right)^{\prime}>0$ for $0<I<\zeta$ and $\left(\frac{I}{f(I)}\right)^{\prime}<0$ for $I>\zeta$.

In epidemiology, these assumptions describe the effects of intervention strategies determining by a critical value $\zeta$ : if $0<I<\zeta$, the incidence rate is increasing, while if $I>\zeta$, the incidence rate is decreasing. Thus we can establish the following SEIR epidemic model:

$$
\left\{\begin{array}{l}
\frac{\mathrm{d} S}{\mathrm{~d} t}=A-\mu S-\frac{\beta I}{f(I)} S, \\
\frac{\mathrm{d} E}{\mathrm{~d} t}=\frac{\beta I}{f(I)} S-(\mu+q) E, \\
\frac{\mathrm{d} I}{\mathrm{~d} t}=q E-(\mu+\delta+\gamma) I, \\
\frac{\mathrm{d} R}{\mathrm{~d} t}=\gamma I-\mu R .
\end{array}\right.
$$

Since $R$ does not appear in the first three equations of system (2), it can be 
reduced to the following three-dimensional system

$$
\left\{\begin{array}{l}
\frac{\mathrm{d} S}{\mathrm{~d} t}=A-\mu S-\frac{\beta I}{f(I)} S, \\
\frac{\mathrm{d} E}{\mathrm{~d} t}=\frac{\beta I}{f(I)} S-(\mu+q) E, \\
\frac{\mathrm{d} I}{\mathrm{~d} t}=q E-(\mu+\delta+\gamma) I .
\end{array}\right.
$$

where the state space is in the first quadrant $R_{+}^{3}=\{(S, E, I): S>0, E>0, I>0\}$.

It follows from system (3) that:

$$
A-(\mu+\delta+\gamma) M \leq \frac{\mathrm{d} M}{\mathrm{~d} t} \leq A-\mu M .
$$

where $M=S+E+I$.

Hence, by integrating the above inequality, there is

$$
\frac{A}{\mu+\delta+\gamma}+\left(M(0)-\frac{A}{\mu+\delta+\gamma}\right) \mathrm{e}^{-(\mu+\delta+\gamma) t} \leq M \leq \frac{A}{\mu}+\left(M(0)-\frac{A}{\mu}\right) \mathrm{e}^{-\mu t} .
$$

Then let $t \rightarrow \infty$, we can get

$$
\frac{A}{\mu+\delta+\gamma} \leq \lim _{t \rightarrow \infty} \inf (M) \leq \lim _{t \rightarrow \infty} \sup (M) \leq \frac{A}{\mu} .
$$

That is

$$
\frac{A}{\mu+\delta+\gamma} \leq S+E+I \leq \frac{A}{\mu}
$$

The feasible region for system (3) is thus a bounded set $\Gamma$ :

$$
\Gamma=(S, E, I) \in R_{+}^{3}: 0<S+E+I \leq \frac{A}{\mu} \subset R_{+}^{3} .
$$

The region $\Gamma$ is a positive invariant set for model (3). Moreover, every trajectory of model (3) eventually stays in a compact subset of $\Gamma$.

\section{Main Results and Proof of Main Results}

In this section, we give main results and the proof of main results.

Notice that model (3) has a disease-free equilibrium point $P_{0}=\left(\frac{A}{\mu}, 0,0\right)$ for all parameter. A main concern of deterministic epidemic model is to find conditions when a disease introduced into a community can develop into a large outbreak, and if it does the disease may become endemic. A useful threshold in this regard for deterministic models is called basic reproduction number $R_{0}$.

Let $X=(E, I, S)^{\mathrm{T}}$, system (3) can be written as [15]

$$
\frac{\mathrm{d} X}{\mathrm{~d} t}=F(X)-V(X),
$$

where 


$$
F(X)=\left(\begin{array}{c}
\frac{\beta I S}{f(I)} \\
0 \\
0
\end{array}\right), V(X)=\left(\begin{array}{c}
(\mu+q) E \\
-q E+(\mu+\delta+\gamma) I \\
-A+\mu S+\frac{\beta I S}{f(I)}
\end{array}\right) .
$$

The jacobian matrices of (9) at the disease-free equilibrium point are

$$
D F\left(P_{0}\right)=\left[\begin{array}{cc}
F & 0 \\
0 & 0
\end{array}\right], D V\left(P_{0}\right)=\left[\begin{array}{cc}
V & 0 \\
J_{1} & J_{2}
\end{array}\right] \text {, }
$$

where

$$
F=\left[\begin{array}{cc}
0 & \frac{\beta A}{\mu f(0)} \\
0 & 0
\end{array}\right], V=\left[\begin{array}{cc}
\mu+q & 0 \\
-q & \mu+\delta+\gamma
\end{array}\right] .
$$

So the next generation matrix of model (3) is

$$
F V^{-1}=\left[\begin{array}{cc}
\frac{\beta A q}{\mu f(0)(\mu+q)(\mu+\delta+\gamma)} & \frac{\beta A}{\mu f(0)(\mu+\delta+\gamma)} \\
0 & 0
\end{array}\right] .
$$

and the spectral radius of $F V^{-1}$ is

$$
\rho\left(F V^{-1}\right)=\frac{\beta A q}{\mu f(0)(\mu+q)(\mu+\delta+\gamma)} .
$$

Therefore the basic reproduction number $R_{0}[16]$ is

$$
R_{0}=\frac{\beta A q}{\mu f(0)(\mu+q)(\mu+\delta+\gamma)} .
$$

The epidemic model (3) has two equilibrium points: one is the disease-free equilibrium point $P_{0}=\left(\frac{A}{\mu}, 0,0\right)$ which exists for all parameter values; and the second is the endemic equilibrium point $P^{*}=\left(S^{*}, E^{*}, I^{*}\right)$ which is a positive solution of the following system

$$
\left\{\begin{array}{l}
A-\mu S^{*}-\frac{\beta I^{*}}{f\left(I^{*}\right)} S^{*}=0 \\
\frac{\beta I^{*}}{f\left(I^{*}\right)} S^{*}-(\mu+q) E^{*}=0 \\
q E^{*}-(\mu+\delta+\gamma) I^{*}=0
\end{array}\right.
$$

From (4),

$$
S^{*}=\frac{(\mu+q)(\mu+\delta+\gamma)}{q \beta} f\left(I^{*}\right), E^{*}=\frac{\mu+\delta+\gamma}{q} I^{*} .
$$

and

$$
A-\frac{(\mu+q)(\mu+\delta+\gamma)}{q} I^{*}-\frac{\mu(\mu+q)(\mu+\delta+\gamma)}{\beta q} f\left(I^{*}\right)=0 .
$$


Set

$$
Q(I)=A-\frac{(\mu+q)(\mu+\delta+\gamma)}{q} I-\frac{\mu(\mu+q)(\mu+\delta+\gamma)}{\beta q} f(I) .
$$

It follows from the assumption (H1) that

$$
Q^{\prime}(I)=A-\frac{(\mu+q)(\mu+\delta+\gamma)}{q}-\frac{\mu(\mu+q)(\mu+\delta+\gamma)}{\beta q} f^{\prime}(I)<0 .
$$

Hence $Q(I)$ is a decreasing function.

Note that

$$
Q(0)=\frac{\mu(\mu+q)(\mu+\delta+\gamma) f(0)}{\beta q}\left(R_{0}-1\right) .
$$

If $R_{0}>1$, we can know that $Q(0)>0$, and because $Q(I)$ is a decreasing function so $Q(I)=0$ has a unique positive solution $I^{*}$, then model (3) has a unique endemic equilibrium $P^{*}=\left(S^{*}, E^{*}, I^{*}\right)$ with

$$
S^{*}=\frac{(\mu+q)(\mu+\delta+\gamma)}{\beta q} f\left(I^{*}\right), E^{*}=\frac{\mu+\delta+\gamma}{q} I^{*} .
$$

So we know that the unique endemic equilibrium point $P^{*}=\left(S^{*}, E^{*}, I^{*}\right)$ of model (3) exists when $R_{0}>1$.

Theorem 3.1. The disease-free equilibrium point $P_{0}=\left(\frac{A}{\mu}, 0,0\right)$ of model (3) is globally asymptotically stable if $R_{0} \leq 1$ or unstable if $R_{0}>1$.

Proof. Define the Lyapunov function

$$
V(S, E, I)=\frac{1}{2}\left(S-\frac{A}{\mu}\right)^{2}+\frac{A}{\mu} E+\frac{A(\mu+q)}{\mu q} I .
$$

Take derivative of $V$ along the solution of model (3), there is

$$
\begin{aligned}
\frac{\mathrm{d} V}{\mathrm{~d} t}= & \left(S-\frac{A}{\mu}\right) \frac{\mathrm{d} S}{\mathrm{~d} t}+\frac{A}{\mu} \frac{\mathrm{d} E}{\mathrm{~d} t}+\frac{A(\mu+q)}{\mu q} \frac{\mathrm{d} I}{\mathrm{~d} t} \\
= & \left(S-\frac{A}{\mu}\right)\left(A-\mu S-\frac{\beta I S}{f(I)}\right)+\frac{A}{\mu}\left(\frac{\beta I S}{f(I)}-(\mu+q) E\right) \\
& +\frac{A(\mu+q)}{\mu q}(q E-(\mu+\delta+\gamma) I) \\
= & -\mu\left(S-\frac{A}{\mu}\right)^{2}-\left(S-\frac{A}{\mu}\right) \frac{\beta I S}{f(I)}+\frac{A}{\mu} \frac{\beta I S}{f(I)}-\frac{A(\mu+q)(\mu+\delta+\gamma)}{\mu q} I \\
= & -\left(\mu+\frac{\beta I}{f(I)}\right)\left(S-\frac{A}{\mu}\right)^{2}+\frac{A}{\mu} \frac{A \beta-\mu(\mu+q)(\mu+\delta+\gamma) \frac{f(I)}{q}}{\mu f(I)} I .
\end{aligned}
$$

Note that $f(I)=f(0)+f^{\prime}(0) I+o(I)$, we have

$$
\begin{aligned}
& A \beta-\mu(\mu+q)(\mu+\delta+\gamma) \frac{f(I)}{q} \\
& =A \beta-\frac{\mu(\mu+q)(\mu+\delta+\gamma)}{q}\left(f(0)+f^{\prime}(0) I+o(I)\right)
\end{aligned}
$$




$$
\begin{aligned}
& \leq A \beta-\frac{\mu(\mu+q)(\mu+\delta+\gamma)}{q} f(0)-\frac{\mu(\mu+q)(\mu+\delta+\gamma)}{q} f^{\prime}(0) I \\
& \leq-\frac{\mu(\mu+q)(\mu+\delta+\gamma) f(0)}{q}\left(1-R_{0}\right)-\frac{\mu(\mu+q)(\mu+\delta+\gamma)}{q} f^{\prime}(0) I \\
& \leq 0 .
\end{aligned}
$$

when $R_{0} \leq 1$.

So

$$
\begin{aligned}
\frac{\mathrm{d} V}{\mathrm{~d} t} \leq & -\left(\mu+\frac{\beta I}{f(I)}\right)\left(S-\frac{A}{\mu}\right)^{2} \\
& -\frac{A}{\mu} \frac{\mu(\mu+q)(\mu+\delta+\gamma)}{q} \frac{f(0)\left(1-R_{0}\right) I+f^{\prime}(0) I^{2}}{\mu f(I)} \\
& <0 .
\end{aligned}
$$

By applying the Lyapunov-LaSall asymptotic stability theorem [17] [18], we conclude that $P_{0}$ is globally asymptotically stable if $R_{0} \leq 1$.

When $R_{0}>1$, the Jacobian matrix of model (3) evaluated at $P_{0}$ is

$$
J\left(P_{0}\right)=\left(\begin{array}{ccc}
-\mu & 0 & -\frac{\beta A}{\mu f(0)} \\
0 & -\mu-q & \frac{\beta A}{\mu f(0)} \\
0 & q & -(\mu+\delta+\gamma)
\end{array}\right),
$$

which has an eigenvalue $-\mu<0$. Denoted by

$$
B=\left(\begin{array}{cc}
-\mu-q & \frac{\beta A}{\mu f(0)} \\
q & -\mu-\delta-\gamma
\end{array}\right) .
$$

We find that when $R_{0}>1$, det $B=\left(1-R_{0}\right)(\mu+q)(\mu+\delta+\gamma)<0$, $\operatorname{tr} B=-(\mu+q+\mu+\delta+\gamma)<0$, so the matrix $B$ must have a positive eigenvalue. Thus, the disease-free equilibrium point $P_{0}$ is unstable whenever $R_{0}>1$. This ends the proof.

Theorem 3.2 If $R_{0}>1$, the unique endemic equilibrium point $P^{*}=\left(S^{*}, E^{*}, I^{*}\right)$ of model (3) is globally asymptotically stable.

Proof. The Jacobia matrix of model (3) evaluated at $P^{*}$ is

$$
J\left(P^{*}\right)=\left(\begin{array}{ccc}
-\mu-\frac{\beta I^{*}}{f\left(I^{*}\right)} & 0 & \frac{(\mu+q)(\mu+\delta+\gamma)\left(I^{*} f^{\prime}\left(I^{*}\right)-f\left(I^{*}\right)\right)}{q f\left(I^{*}\right)} \\
\frac{\beta I^{*}}{f\left(I^{*}\right)} & -\mu-q & -\frac{(\mu+q)(\mu+\delta+\gamma)\left(I^{*} f^{\prime}\left(I^{*}\right)-f\left(I^{*}\right)\right)}{q f\left(I^{*}\right)} \\
0 & q & -\mu-\delta-\gamma
\end{array}\right) .
$$


The characteristic polynomial of $J\left(P^{*}\right)$ is $\lambda^{3}+c_{1} \lambda^{2}+c_{2} \lambda+c_{3}=0$, where

$$
\left\{\begin{array}{l}
c_{1}=3 \mu+q+\delta+\gamma+\frac{\beta I^{*}}{f\left(I^{*}\right)}>0, \\
c_{2}=\left(\mu+\frac{\beta I^{*}}{f\left(I^{*}\right)}\right)(2 \mu+q+\delta+\gamma)+\frac{(\mu+q)(\mu+\delta+\gamma) f^{\prime}\left(I^{*}\right)}{f\left(I^{*}\right)} I^{*}>0, \\
c_{3}=\frac{\left(\beta+\mu f^{\prime}\left(I^{*}\right)\right)(\mu+q)(\mu+\delta+\gamma)}{f\left(I^{*}\right)} I^{*}>0 .
\end{array}\right.
$$

Clearly $c_{1} c_{2}-c_{3}>0$. Therefore, by the Routh-Hurwitz criterion we can conclude that $P^{*}$ is locally asymptotically stable.

Next, we need to prove that $P^{*}$ is globally asymptotically stable. Define a Lyapunov function

$$
V(S, E, I)=\left|S-S^{*}\right|+\left|E-E^{*}\right|+\left|I-I^{*}\right| .
$$

Obviously, $V\left(P^{*}\right)=0$, and when $P \neq P^{*}, V(P) \neq 0$. Remember that $P^{*}$ is the solution of system (3), the upper right derivative of $V$ can be estimated:

$$
\begin{aligned}
D^{+} V= & \operatorname{sgn}\left(S-S^{*}\right)\left(S-S^{*}\right)+\operatorname{sgn}\left(E-E^{*}\right)\left(E-E^{*}\right)+\operatorname{sgn}\left(I-I^{*}\right)\left(I-I^{*}\right) \\
= & \operatorname{sgn}\left(S-S^{*}\right)\left(A-\mu S-\frac{\beta I S}{f(I)}-\left(A-\mu S^{*}-\frac{\beta I^{*} S^{*}}{f\left(I^{*}\right)}\right)\right) \\
& +\operatorname{sgn}\left(E-E^{*}\right)\left(\frac{\beta I S}{f(I)}-(\mu+q) E-\left(\frac{\beta I^{*} S^{*}}{f\left(I^{*}\right)}-(\mu+q) E^{*}\right)\right) \\
& +\operatorname{sgn}\left(I-I^{*}\right)\left(q E-(\mu+\delta+\gamma) I-\left(q E^{*}-(\mu+\delta+\gamma) I^{*}\right)\right),
\end{aligned}
$$

where

$$
\operatorname{sgn}(x)= \begin{cases}-1, & x<0 \\ 0, & x=0 \\ 1, & x>0 .\end{cases}
$$

In (5), there are 8 kinds of situations for the size of $S$ and $S^{*}, E$ and $E^{*}, I$ and $I^{*}$. It is enough to analyze the situation of $S>S^{*}, E>E^{*}$ and $I>I^{*}$ since the other situations are similar.

Firstly there is

$$
\begin{aligned}
D^{+} V & \leq-\mu\left|S-S^{*}\right|-\mu\left|E-E^{*}\right|-(\mu+\delta+\gamma)\left|I-I^{*}\right| \\
& <-\mu\left|S-S^{*}\right|-\mu\left|E-E^{*}\right|-\mu\left|I-I^{*}\right| \\
& <-\mu V .
\end{aligned}
$$

Integrating from $t_{0}$ to $t$ on both sides of (6), we have

$$
V(t)+\mu \int_{t_{0}}^{t} V \mathrm{~d} t \leq V\left(t_{0}\right)<+\infty .
$$

Since the front set $\Gamma$ has a boundary, $S, E$ and $I$ must have boundaries, and 
their derivatives are bounded, which means that $V$ is uniformly continuous.

By Barbalat Lemma, there is $\lim _{t \rightarrow \infty} V(t)=0$, so

$$
D^{+} V<-\mu V<0 .
$$

Thus, the unique endemic equilibrium point $P^{*}$ of model (3) is globally asymptotically stable. This completes the proof.

\section{Applications and Numerical Simulations}

In this section, we choose the function $f(I)$ as

$$
f(I)=1+\alpha^{2} \text {. }
$$

which was proposed by Xiao and Ruan [5].

Then the model(3) becomes

$$
\left\{\begin{array}{l}
\frac{\mathrm{d} S}{\mathrm{~d} t}=A-\mu S-\frac{\beta I}{1+\alpha^{2}} S, \\
\frac{\mathrm{d} E}{\mathrm{~d} t}=\frac{\beta I}{1+\alpha^{2}} S-(\mu+q) E, \\
\frac{\mathrm{d} I}{\mathrm{~d} t}=q E-(\mu+\delta+\gamma) I .
\end{array}\right.
$$

It is easy to verify that the function $f(I)=1+\alpha^{2}$ satisfies assumption $H(1)$ and $H(2)$, at the same time,

$$
R_{0}=\frac{\beta A q}{\mu(\mu+q)(\mu+\delta+\gamma)} .
$$

The model (7) has a disease-free equilibrium point $P_{0}=\left(\frac{A}{\mu}, 0,0\right)$ and an endemic equilibrium point $P^{*}=\left(S^{*}, E^{*}, I^{*}\right)$ with

$$
\left\{\begin{array}{l}
S^{*}=\frac{\mu(\mu+q)(\mu+\delta+\gamma)}{\beta q}\left(1+\alpha^{* 2}\right), \\
E^{*}=\frac{\mu+\delta+\gamma}{q} I^{*}, \\
I^{*}=\frac{\sqrt{\beta^{2}+4 \alpha \mu^{2}\left(R_{0}-1\right)}-\beta}{2 \mu \alpha},
\end{array}\right.
$$

when $R_{0}>1$.

Using the arguments in section 3, we can obtain the following results.

Theorem 4.1 The disease-free equilibrium point $P_{0}$ of (7) is globally asymptotically stable if $R_{0} \leq 1$ and unstable if $R_{0}>1$.

Theorem 4.2 The endemic equilibrium point $P^{*}$ of $(7)$ is globally asymptotically stable if $R_{0}>1$.

We know that $\mu, \beta, q, \delta, \gamma$ are greater than 0 and less than 1 , so we selected some data for numerical simulation. After many numerical simulation experiments, we have selected the following data with good interpretation.

Let $A=1, \quad \mu=0.15, \quad \beta=0.2, q=0.1, \delta=0.05, \quad \gamma=0.1, \quad \alpha=0.01$ in 
Figure 1, we have $R_{0}=1.333>1$. As is shown in Figure 1 , the curve $S$ starts to go up very quickly, goes down at a certain value and then tends stabilize. The curves $\mathrm{E}$ and I go up at a certain value and flatten out. This suggests that the disease is present. Figure 1 shows that when $R_{0}>1$, the endemic equilibrium point $P^{*}$ exists and is globally asymptotically stable, which is consistent with the previous conclusion.

Next, we choose some other parameter values: $A=1, \mu=0.2, \beta=0.2$, $q=0.1, \delta=0.05, \gamma=0.1, \alpha=0.01$. We have $R_{0}=0.9523<1$. As shown in Figure 2, the curve $S$ rises rapidly, reaches a certain value and then flattens out. While the curves $\mathrm{E}$ and I monotonically decrease and go to zero which indicate that the disease disappears over time. Figure 2 reflects that the disease dies out and the disease-free equilibrium point $P_{0}$ is globally asymptotically stable.

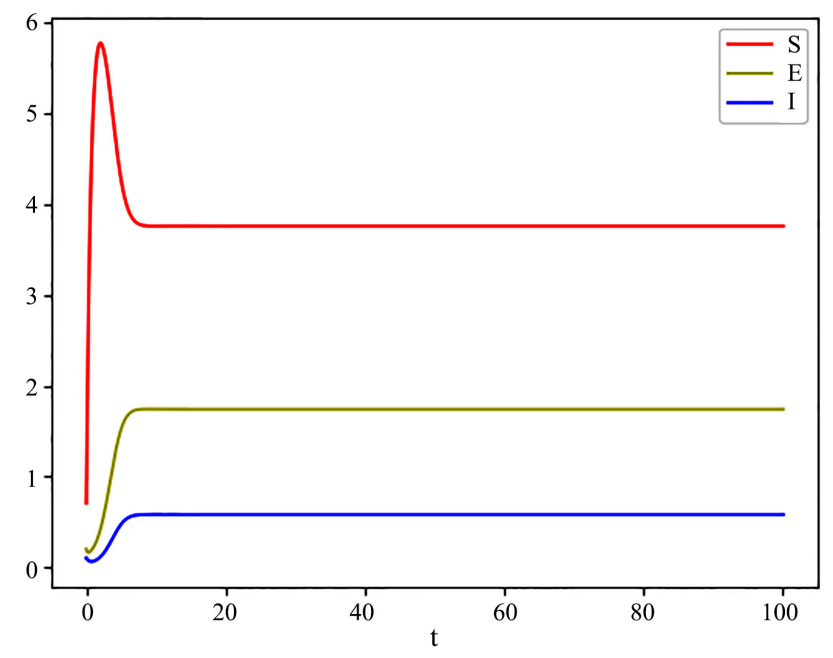

Figure 1. The path of $S(t), E(t), I(t)$ for the model (7) with initial values $(0.7,0.2,0.1), R_{0}=1.333>1$.

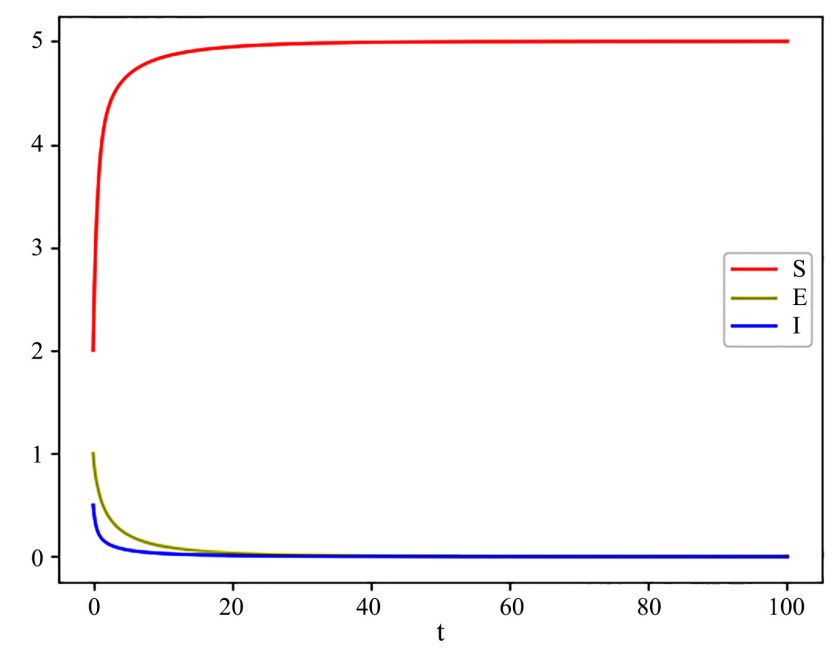

Figure 2. The path of $S(t), E(t), I(t)$ for the model (7) with initial values $(2,1,0.5), R_{0}=0.953<1$. 


\section{Brief Summary}

In this paper, we consider the global stability of an SEIR epidemic model with infection force under intervention strategies. We suppose that the infection force can be factorized into $\frac{\beta I}{f(I)}$, where $f(I)$ satisfies some conditions, and we use the regeneration matrix to obtain the basic reproductive number $R_{0}$. We also proved the existence of the equilibrium point.

We prove that if $R_{0} \leq 1$, there exists only the disease-free equilibrium point which is globally asymptotically stable; if $R_{0}>1$, there is a unique endemic equilibrium point and the endemic equilibrium point is globally asymptotically stable.

\section{Conflicts of Interest}

The authors declare no conflicts of interest regarding the publication of this paper.

\section{References}

[1] Kermack, G.W.O. and Mckendrick, A.G. (1937) Contributions to the Mathematical Theory of Epidemics IV. Analysis of Experimental Epidemics of the Virus Disease Mouse Ectromelia. Epidemiology \& Infection, 37, 172-187. https://doi.org/10.1017/S0022172400034902

[2] Cai, Y., Kang, Y., Banerjee, M. and Wang, W. (2015) A Stochastic Sirs Epidemic Model with Infectious Force under Intervention Strategies. Journal of Differential Equations, 259, 7463-7502. https://doi.org/10.1016/j.jde.2015.08.024

[3] Ma, Z., Zhou, Y. and Wu, J. (2009) Modeling and Dynamics of Infectious Diseases. Higher Education Press, Beijing.

[4] Wang, W. (2017) Epidemic Models with Nonlinear Infection Forces. Mathematical Biosciences \& Engineering, 3, 267-279. https://doi.org/10.3934/mbe.2006.3.267

[5] Xiao, D. and Ruan, S. (2007) Global Analysis of an Epidemic Model with NonMonotone Incidence Rate. Mathematical Biosciences, 208, 419-429.

https://doi.org/10.1016/j.mbs.2006.09.025

[6] Cui, J.A., Tao, X. and Zhu, H. (2008) An Sis Infection Model Incorporating Media Coverage. Rocky Mountain Journal of Mathematics, 38, 1323-1334. https://doi.org/10.1216/RMJ-2008-38-5-1323

[7] Cui, J., Sun, Y. and Zhu, H. (2008) The Impact of Media on the Control of Infectious Diseases. Journal of Dynamics \& Differential Equations, 20, 31-53. https://doi.org/10.1007/s10884-007-9075-0

[8] Tchuenche, J.M. and Bauch, C.T. (2012) Dynamics of an Infectious Disease Where Media Coverage Influences Transmission. ISRN Biomathematics, 2012, Article ID: 581274. https://doi.org/10.5402/2012/581274

[9] Xiao, Y., Zhao, T. and Tang, S. (2013) Dynamics of an Infectious Diseases with Media/Psychology Induced Non-Smooth Incidence. Mathematical Biosciences \& Engineering, 10, 445-461. https://doi.org/10.3934/mbe.2013.10.445

[10] Tang, S., Xiao, Y., Yuan, L., Cheke, R.A. and Wu, J. (2012) Campus Quarantine (Fengxiao) for Curbing Emergent Infectious Diseases: Lessons from Mitigating a/h1n1 in Xi'an, China. Journal of Theoretical Biology, 295, 47-58. 
https://doi.org/10.1016/j.jtbi.2011.10.035

[11] Khan, M.A., Rahman, M., Khanam, P.A., Khuda, B., Kane, T.T. and Ashraf, A. (1997) Awareness of Sexually Transmitted Disease among Women and Service Providers in Rural Bangladesh. International Journal of Std \& Aids, 8, 688-696. https://doi.org/10.1258/0956462971919066

[12] Xiao, Y., Tang, S. and Wu, J. (2015) Media Impact Switching Surface during an Infectious Disease Outbreak. Scientific Reports, 5, Article No. 7838. https://doi.org/10.1038/srep07838

[13] Ruan, S.G. and Wang, W.D. (2003) Dynamical Behavior of an Epidemic Model with a Nonlinear Incidence Rate. Journal of Differential Equations, 188, 135-163. https://doi.org/10.1016/S0022-0396(02)00089-X

[14] Cai, Y., Kang, Y. and Wang, W. (2017) A Stochastic Sirs Epidemic Model with Nonlinear Incidence Rate. Applied Mathematics \& Computation, 305, 221-240. https://doi.org/10.1016/j.amc.2017.02.003

[15] Van den Driessche, P. and Watmough, J. (2002) Reproduction Numbers and Sub-Threshold Endemic Equilibria for Compartmental Models of Disease Transmission. Mathematical Biosciences, 180, 29-48. https://doi.org/10.1016/S0025-5564(02)00108-6

[16] Bai, Z.G. (2013) Basic Reproduction Number of Periodic Epidemic Models. Chinese Journal of Engineering Mathematics, 30, 175-183.

[17] LaSalle, J.P. (1976) The Stability of Dynamical Systems. CBMS-NSF Regional Conference Series in Applied Mathematics. Book 27, Society for Industrial and Applied Mathematics, Philadelphia, 1121-1130.

[18] Lyapunov, A.M. (1992) The General Problem of the Stability of Motion. International Journal of Control, 55, 531-534. https://doi.org/10.1080/00207179208934253 